The wbristied manuscript has been authored by controctor of the U.S. Governmint under controct No. W.31.109-ENG-38. Accordingly, the U.S. Gowernment retains: Accordingly, tho U.S. Government retains : nonexclusive, royalty.free license to publish or reproduce the published formt of this contribution, or nllow others to do $\%$, for U. S. Government purposes. $\operatorname{conf}-900924 \cdot 10$

ANL-HEP-CP-90-101

For the Proceedings of the

9th International Symposium on

High Energy Spin Physics

Bonn, West Germany

10-14 September 1990

\section{EXPERIMENTS WITH FERMILAB POLARIZED PROTON AND ANTIPROTON BEAMS $§$}

(For the Fermilab E-704 Collaboration ")

ANL-HEP-CP--90-101

A. Yokosawa

DE9 1006551

High Energy Physics Division, Argonne National Laboratory Argonne, Illinois 60439 USA

\begin{abstract}
We summarize activities concerning the Fermilab polarized beams. They include a brief description of the polarized-beam facility, measurements of beam polarization by polarimeters, asymmetry measurements in the $\pi^{\circ}$ production at high $P_{\perp}$ and in the $\Lambda\left(\Sigma^{0}\right), \pi^{ \pm}, \pi^{0}$ production at large $x_{F}$, and $\Delta \sigma_{L}(p p, \overrightarrow{p p})$ measurements.
\end{abstract}

\title{
Introduction
}

The Eermilab polarized-beam facility at $200 \mathrm{GeV} / \mathrm{c}$ became operational in 1987. At that time, we mainly concentrated on the measurements of beam polarization by polarimeters and on the studies of beam polarization monitors. Recently we carried out the polarized-beam program designated as $\mathrm{E}-704$ by ar. international collaboration.

We summarize these activities including the discussions of some preliminary data on $P_{\perp}$ and $x_{F}$ dependence.

\section{Polarized Beam Facility}

The Fermilab polarized-beam facilityl was operated during the past TeV-II (fixed target) period which ended in February 1988.

An axtracted beam from the Tevatron is delivered through the MP primarybeam 1 ine to the Meson Detector Building where a 0.73 -interaction-length Be target is utilized to produce $\Lambda$ and $\bar{\Lambda}$ at $\theta_{\mathrm{c} \cdot \mathrm{m} .} \approx 0^{\circ}$. Protons and antiprotons from the $\Lambda$ and $\bar{\Lambda}$ decays respectively are brought to a final target position in the MP hall through the MP secondary beam $(200 \mathrm{CeV} / \mathrm{c})$ line. 
Polarized protons from the virtual sources as shown in Fig. 1 are focussed in the tagging section, where both the momentum and polarization are selected. 1

The typical beam $f l u x(\Delta p / p= \pm 5 \%)$ for $3 \times 10^{12}$ incident protons per 20sec spill at $200 \mathrm{GeV} / \mathrm{c}$ were: ( $\mathrm{P}_{\text {av }}$ is average polarization)

$\begin{array}{lccc} & \begin{array}{l}\text { Tagged Beam } \\ \left.P_{\text {av }}=45 \%\right)\end{array} & \begin{array}{l}\text { Total Protons } \\ \text { (antiprotons) }\end{array} & \text { Background } \pi^{1} s \\ \text { Protons } & 1.0 \times 10^{7} & 2.0 \times 10^{7} & 2.0 \times 10^{6} \\ \text { Antiprotons } & 5.0 \times 10^{5} & 1.0 \times 10^{6} & 5.0 \times 10^{6}\end{array}$

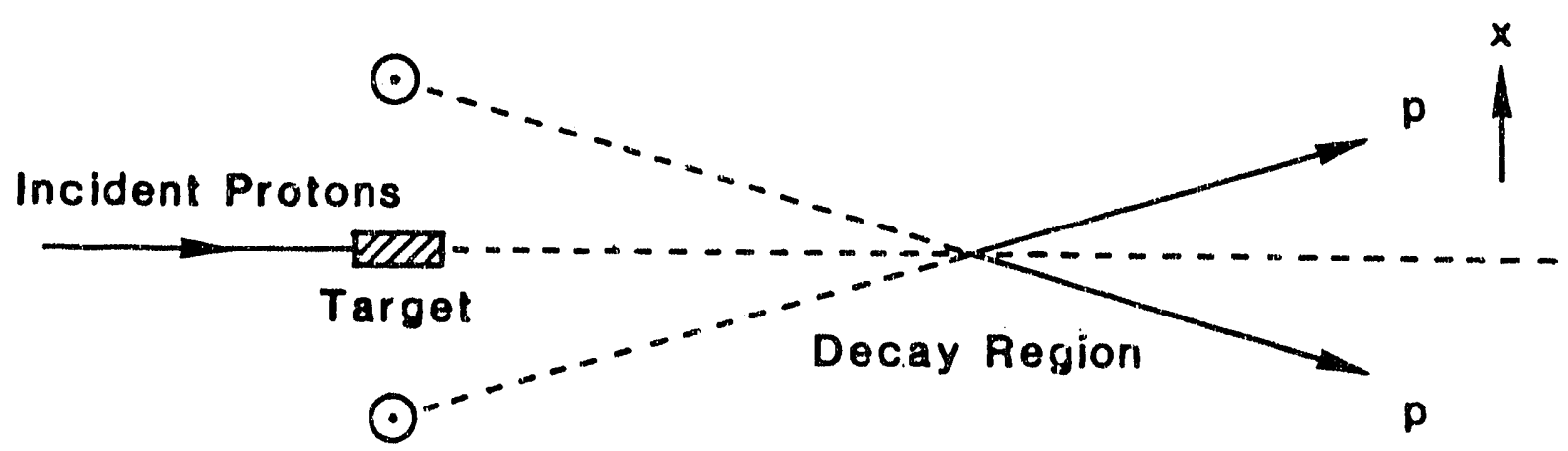

\section{Virtual Sources}

Figure 1 Virtual sources (top view).

\section{Primak of f-Ef fect Measurement}

The asymmetry of the nuclear coherent Coulomb $\pi^{\circ}$ production process ("Primakoff process"), was measured ${ }^{2}$ for the first time with the use of the polarized-proton beam. The apparatus consisted of a lead-glass calorimeter for $\pi^{\circ}$ detection and a magnetic spectrometer for the scattered protons. A large asymmetry in the region of $\left|t^{\prime}\right|<0.001(\mathrm{GeV} / \mathrm{c})^{2}$ and $1.36<M\left(\pi^{\circ} \mathrm{p}\right)<$ $1.52 \mathrm{GeV} / \mathrm{c}^{2}$ was observed for the reaction $\mathrm{p}+\mathrm{Pb}+\mathrm{p}+\pi^{\circ}+\mathrm{Pb}$, where the Coulomb process is predominant. The expected null asymmetry was observed in the larger $\left|t^{\prime}\right|$ region where the diffractive-dissociation process is predominant. 
The observed $\phi$-angle dependence of the coherent $\pi^{\circ}$ production process may be expressed as $1+\left(f T(\theta) P_{B}\right) \cos \phi$, where $T(\theta)$ is the analyzing power (target azimuthal asymmetry) for photoproduction of $\pi^{\circ}$ from a polarized proton target at c.m. polar angle $\theta, \phi$ is the azimuthal angle, $P_{B}$ is the transverse polarization, and the parameter $f$ is a dilution factor caused by the diffractive dissociation. The raw asymmetry at $\phi$ is obtained as

$$
A(\phi)=\left(N^{\dagger}(\phi)-N^{\dagger}(\phi)\right) /\left(N^{\dagger}(\phi)+N^{\dagger}(\phi)\right)=f T(\theta) P_{B} \cos \phi=\varepsilon \cos \phi,
$$

where $\mathrm{N}^{\dagger}(\phi)$ and $\mathrm{N}^{+}(\dot{\psi})$ are the number of events at $\phi$ for the up and down spin direction of the incident proton, respectively.

The measured asymmetry for the Coulomb process is consistent with the analyzing power (about $-70 \%$ ) of the $\pi^{\circ}$ production process deduced from existing low-energy $r+p+\pi^{\circ}+p$ data. The results demonstrate that the Primakoff process is useful for the measurement of proton and antiproton polarization at high energy.

\section{Coulomb-Nuclear Interference Measurements}

The analyzing power, $A_{W}$, of proton-proton, proton-hydrocarbon, and ant iproton-hydrocarbon scattering in the Coulomb-nuclear region was measured with use of the polarized-proton and polarized-antiproton beams. ${ }^{3}$ For the elastic scattering at small $|t|$, a set of scintillation counters was utilized to detect the recoil proton which stops within a very short range in the scintillator. The results at $|t| \sim 0.003(\mathrm{GeV} / \mathrm{c})^{2}$ show the value $\mathrm{A}_{\mathrm{N}}=(2.4 \pm 0.9) \%$ with the polarized-proton beam, and $A_{N}=(-4.6 \pm 1.9) \%$ with the polarizedantiproton beam both on a hydrocarbon target, and also $A_{N}=(4.5 \pm 2.8) \%$ of proton-proton scattering. These results are consistent with predictions ${ }^{4-6}$ based on Coulomb-nuclear interference. Recently $A_{N}$ measurements were repeated with much higher statistics than those mentioned above. 
Single-spin Asymmetxy in $p^{4} p \rightarrow x^{\circ} X$ and $\bar{p}^{4} p \rightarrow \pi^{\circ} X$ at $H i g h P_{\perp-}$

This experiment is recently completed at incident proton momentum of 200 $\mathrm{GeV} / \mathrm{c}$. Preliminary data of the $\mathrm{p}^{\uparrow} \mathrm{p}$ reaction show that the asymmetry values $\left(A_{N}\right)$ at $x_{F}=0$ are approximately zero (or small negative) up to $p_{\perp}=3.5 \mathrm{GeV} / \mathrm{c}$ and then begin to rise to $\sim 40 \%$ in the region of $\mathrm{p}_{\perp}=4$ to $5 \mathrm{GeV} / \mathrm{c}$ as shown in Fig. 2. At lower energies as seen in the $\mathrm{BNL}^{7}\left(\mathrm{p}^{\dagger} \mathrm{p}+\pi^{+} \mathrm{X}\right), \mathrm{CERN}^{8}$

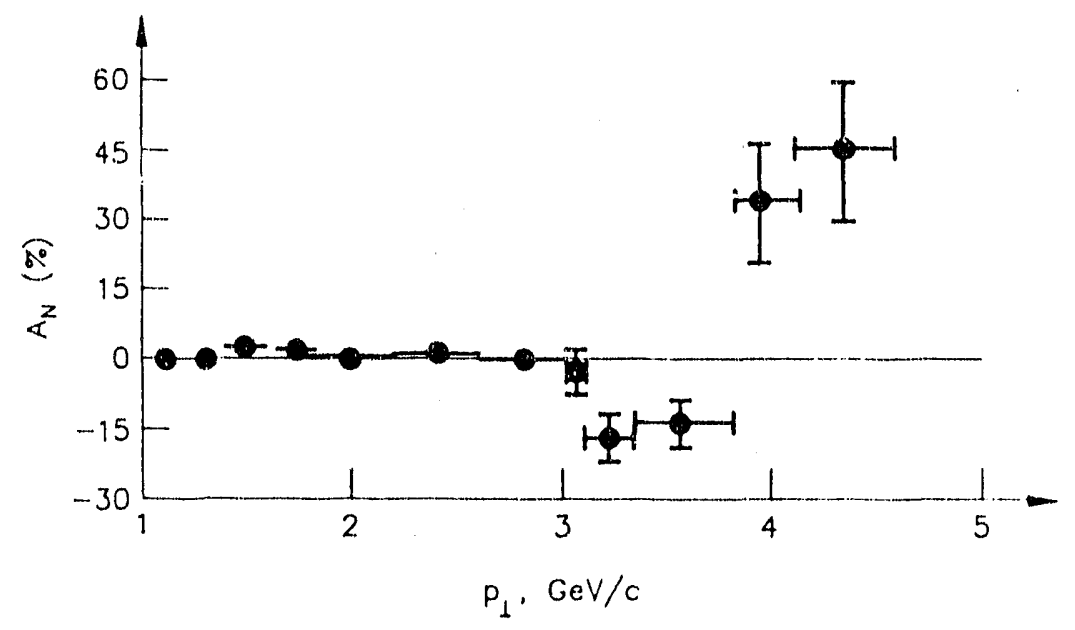

Fig. $2 p_{1}$ dependence of $A_{N}$ at $x_{F} \approx 0$ (preliminary data).

$\left(p p^{\uparrow}+\pi^{\circ} x\right)$, Serpukhov $9\left(\pi^{-} p^{\dagger}+\pi^{\circ} x\right)$ data, this rapid rise from zero to large positive values,,$"$ was also observed as shown in Fig. 3 although none of the data exceetled $P_{\perp}=3 \mathrm{GeV} / \mathrm{c}$. A new finding is that all the $A_{N}$ data of $\pi^{\circ}$ or $\pi^{+}$ production at $x_{F} \approx 0$ show the large positive asymetries begin at $x_{\perp}=0.4$ in the region $\sqrt{s}=5$ to $20 \mathrm{GeV}$ as show in Fig. 4. This is strong indication that we are indeed observing asymmetries caused by hard scattering. We note that the common crossing point $x_{1}=0.4$ was pointed out in the Ref. 9 in the

* Data taken with polarized targets (Refs. 8 and 9) are normalized to the measurements with polarized beams, that is, the sign of $A_{N}$ (hp ${ }^{\dagger} \rightarrow h x$, where $h$ represents hadron) is reversed. Note that $A_{N}(p h+h X)=-A_{N}$ $\left(h p^{\uparrow}+h X\right)$ at $x_{F} \approx 0$. 


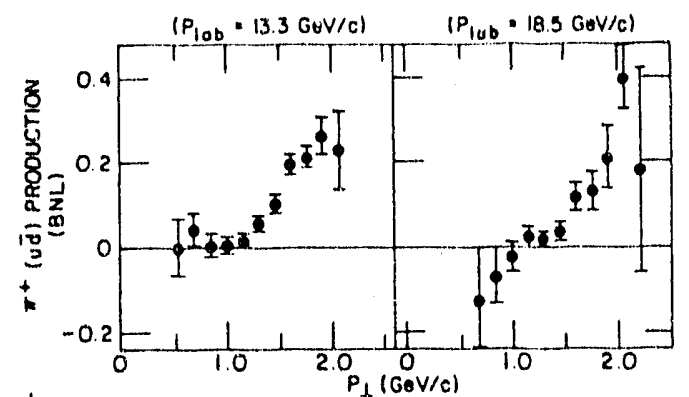

region $\sqrt{\mathrm{s}}=5$ to $8.5 \mathrm{GeV} / \mathrm{c}$. Theoretically single-spin asymmetries are discussed within the context of the QCD hardscattering model. 10 By knowing the quark content of $\pi^{+}=u \bar{d}$ and $\pi^{0}=(u \bar{u}-d \bar{d}) / \sqrt{2}$, polarized u quark in the polarized proton beam is considered $\mathrm{t}$, be the career of the spin information.

Figure 3

Asymmetry $A_{N}(\%)$ vs. $p_{\perp}$ at $p_{1 a b}=1.33$ to $40 \mathrm{GeV} / \mathrm{c}$

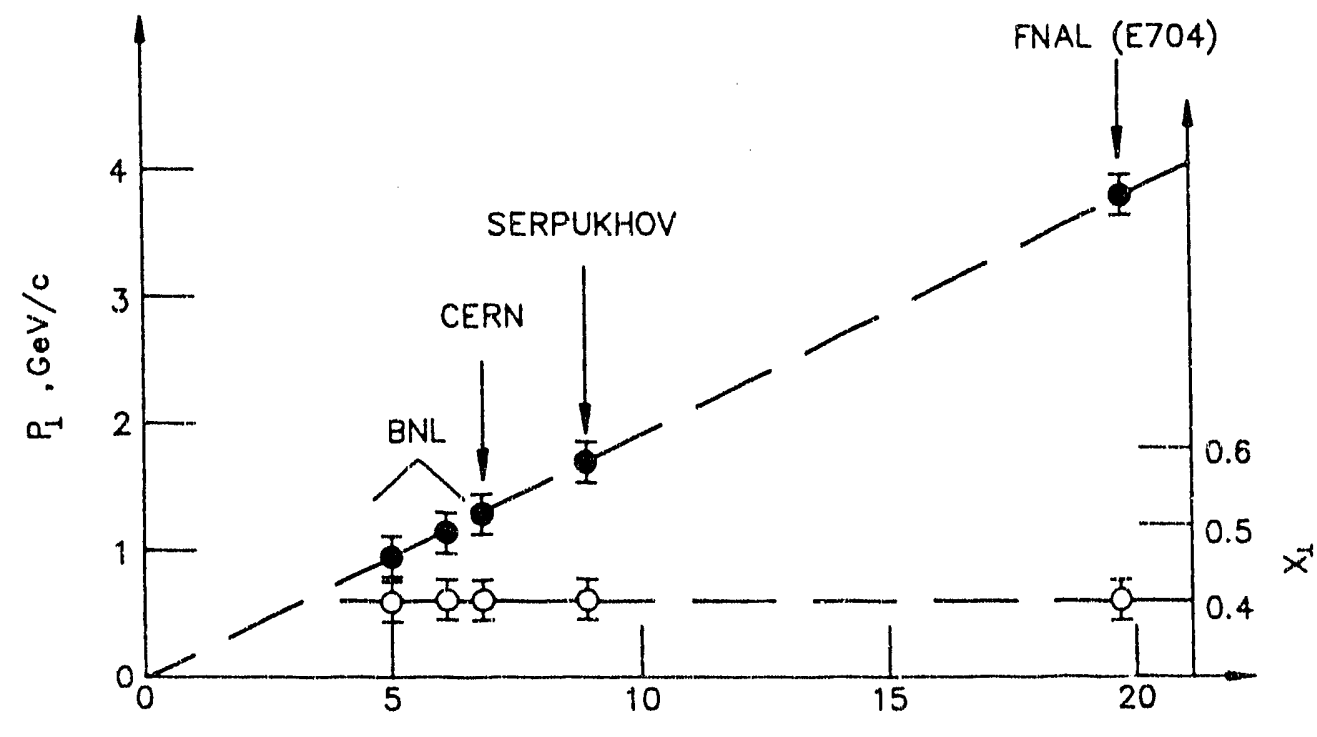

$\sqrt{S}, \mathrm{GeV}$

Figure $4 \mathrm{P}_{\mathrm{T}}$ and $\mathrm{x}_{\mathrm{T}}$ positions at $\mathrm{A}_{\mathrm{N}}=0 \rightarrow$ large positive vs. $\sqrt{\mathrm{s}}$. 
Single-spin asymmetry in $\overline{\mathrm{p}}^{\dagger} \mathrm{p}+\pi^{0 \mathrm{X}}$ shows a similar $\mathrm{p}_{\perp}$ dependendce as the $p^{\uparrow} p$ case. However, data are limited only up to $p_{1}=3.5 \mathrm{GeV} / \mathrm{c}$. $\underline{x}_{F}$ Dependence of Single-Spin Asymmetry in $\mathrm{p}^{4} \mathrm{p} \rightarrow \pi^{\circ} \mathrm{X}$ and $\overline{\mathrm{p}}^{+} \mathrm{p} \rightarrow \pi^{\circ} \mathrm{X}$ Measurements on the $x_{F}$ dependence at $200 \mathrm{GeV} / \mathrm{c}$ covering $\mathrm{p}_{\perp}$ up to $2 \mathrm{GeV} / \mathrm{c}$ were recently completed. Asymmetry values in the $\mathrm{p}^{\dagger} \mathrm{p}$ reaction are consistent with zero up to $\mathrm{x}_{\mathrm{F}}=0.3$ to 0.4 , and then linearly increase to $+20 \%$ near $\mathrm{x}_{\mathrm{F}}=$ 1.0 as shown in Fig. 5. The data suggest an influence of polarized u quarks at large $x_{F}$. Also they are consistent with earlier datall taken at $\left\langle x_{F}\right\rangle=$ 0.52. This is the first $x_{F}$ dependence data ever obtained in the $\pi$ production. It is interesting to notice that our data resembled $x_{F}$ dependnece for $\Lambda$ polarization in $p p \rightarrow \Lambda^{\uparrow} X$ where polarized $s$ quarks were considered ${ }^{12}$ to be responsible for high polarization.

Single-spin asymmetry in $\overline{\mathrm{P}}^{\uparrow} \mathrm{P}+\pi^{\circ} \mathrm{X}$ shows a similar $\mathrm{x}_{F}$ dependence as $\mathrm{P}^{\uparrow} \mathrm{P}$ case.

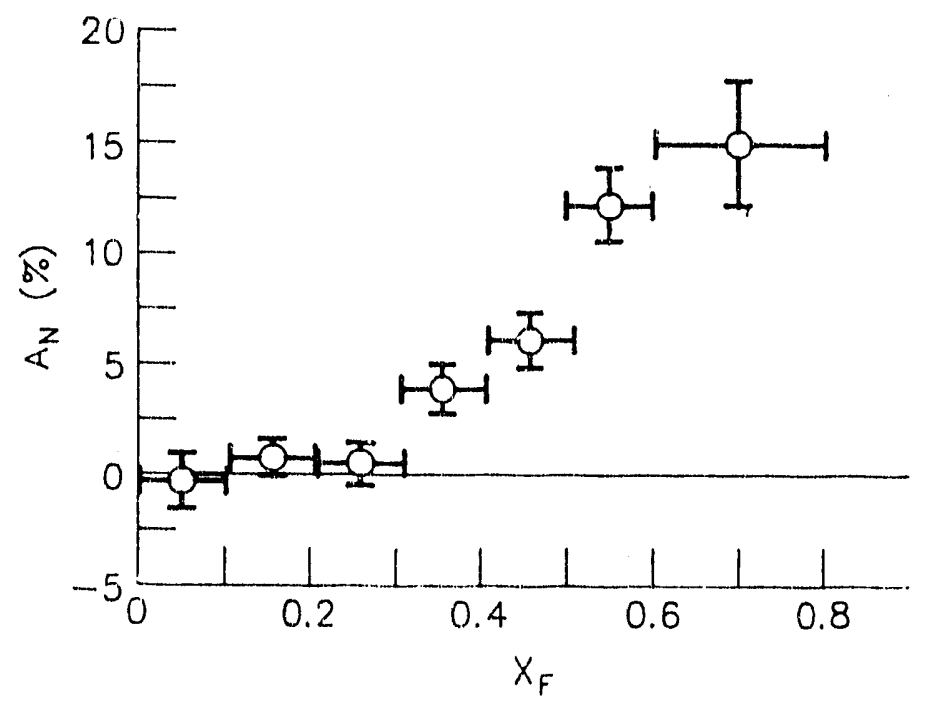

Figure $5 \mathrm{x}_{\mathrm{F}}$ dependence of $\mathrm{A}_{\mathrm{N}}$ at $\mathrm{P}_{\mathcal{L}}=0.5$ to $2.0 \mathrm{GeV} / \mathrm{c}$ (preliminary data).

\section{A Short Summary on $\mathbf{x}_{\perp}$ and $\mathbf{x}_{F}$ Dependence}

There are two distinct common phenomena observed in the $x_{\perp}$ and $x_{F}$ dependence of the one-spin asymmetries. The asymmetry values are zero or small for both $x_{\perp}$ and $x_{F}<0.3$ to 0.4 . Then there is a rise from zero to 
large positive values for $x_{\perp}$ and $x_{F}>0.3$ to 0.4 . It will be interesting to find out if these two phenomena are related. One strong hint is that polarized u quarks seem responsible to the rise in $A_{N}$ at high $x_{\perp}$ and high $x_{F}$. It is interesting to investigate if these phenomena may be related to the origin of proton spin. High- $\mathrm{P}_{\perp}$ and nigh $-\mathrm{x}_{\mathrm{F}}$ scattering phenomena were interpreted as an indication for the existence of rotating color charges in polarized protons. 13

${ }_{0} \mathrm{~L}$ ( $\mathrm{PP}$ and $\overline{\mathrm{p}}$ ), and Double-Spin Asymetry in $\mathrm{p}^{+} \mathrm{p}^{+} \rightarrow \pi^{\circ} \mathrm{X}$ and $\overline{\mathrm{P}}^{+} \mathrm{p}^{+} \rightarrow \pi^{\circ} \mathrm{X}$ Difference in total cross sections for pure spin states, $\Delta \sigma_{L}$ ( $p p$ and $\bar{p} p$ ), was simultaneously measured at $200 \mathrm{GeV} / \mathrm{c}$ with the $\pi^{\circ}$ production up to $\mathrm{P}_{\perp}=3$ $\mathrm{GeV} / \mathrm{c}$. Data are currently being analyzed.

\section{$\underline{\pi^{ \pm}}$and Hyperon Production on Hydrogen Target With Polarized Beam}

Measurements of $\mathrm{p}^{\dagger} \mathrm{p} \rightarrow\left(\pi^{ \pm}, \Lambda^{\circ}, \Sigma^{\circ}\right)+\mathrm{X}$ at $200 \mathrm{GeV} / \mathrm{c}$ were completed and data are currently being analyzed.

\section{Future Experiments}

We have proposed the following simultaneous measurements using a polarized beam and a polarized target.

i) Spin dependence in direct-gamma production at high $\mathrm{p}_{1}$ To understand the basic question of the origin of proton spin, we may be able to determine the gluon spin distribution in the proton by measuring the spin correlation parameter $A_{L L}$ in the direct-r production at high $\mathrm{p}_{\perp}$, with longitudinally polarized protons on longitudinally polarized target nucleons.

$$
A_{L L}=\left(1 / P_{B} P_{T}\right) \frac{N(\stackrel{+}{*})-N(\stackrel{+}{+})}{N(\underset{+}{+})+N(+)}
$$

where $P_{B}$ is beam polarization, $P_{T}$ is target polarization, and arrows indicate the spin direction in the laboratory system. 
The QCD Compton effect, "gluon + quark + gamma + quark", is expected to be the dominant mechanism for direct-r production at large $p_{\perp}$. The parameter $A_{L L}$ is approximately proportional to the gluon polarization. 14,15

Our plan is to carry out the $A_{L L}$ measurements up to $p_{\perp}=5 \mathrm{GeV} / \mathrm{c}$ with reasonable statistical accuracy. Our main detectors consist of lead-glass counters and proportional wire chambers.

ii) Spin dependence of $x^{2}(3555)$ production.

We plan to measure the spin dependence of $x^{2}$ (3555) production, which will be simultaneously carried out with the above mentioned direct-gamma measurements. The double-spin asymmetry, $A_{L L}$, in $p^{\uparrow} p^{\uparrow} \rightarrow x^{2}(3555)+J / \psi+\gamma$ is also expected to provide a means to study the spin dependent gluon structure function. The $15 \%$ decay branching ratio of $x^{2}(3555)$ to $\mathrm{J} / \psi+\gamma$ allows us to analyze the helicity of the charmonium state.

There is general agreement $t^{16}$ theoretically that the $x^{2}$ (3555) state is mainly produced by gluon-gluon fusion as shown below and there are promising experimental results ${ }^{18}$ suggesting that simple gluon fusion is sufficient to account for the $x^{2}$ (3555) production in proton interactions at 200 and $250 \mathrm{GeV} / \mathrm{c}$. The measured two-spin asymmetries (as defined below) give information on the initial gluon polarization, which can be used to reconstruct the gluon spin distribution in the polarized proton. ${ }^{18}$ By considering the fusion process, if the initial helicity state is (+-), that is $(\ddagger)$, the $J_{z}=2$ and this state produces $\chi^{2}$.

To be exact, the observable $A_{L L}$ is related to the distribution function of a polarized gluon in a polarized proton expressed as $G_{+}(x)$ and $G_{-}(x)$ with same- and opposite-sign helicities respectively. 18

$$
A_{L L}\left(x_{F}\right)=(1-R) /(1+R)\left[\frac{\Delta G}{G}\left(x_{1}\right) \cdot \frac{\Delta G}{G}\left(x_{2}\right)\right]
$$


where $x_{1}, x_{2}$, and $x_{F}$ are the longitudinal-momentum fraction of gluons, $R$ is the ratio of matrix elements $f_{+}\left(f_{-}\right)$which are the squared matrix elements for the production of $x^{2}$ out of two gluons with same-(opposite-) sign helicities, and $\Delta G / G(x) \equiv\left(C_{+}(x)-C_{-.}(x)\right) /\left(G_{+}(x)+G_{-}(x)\right)$.

Theoretical predictions were recently made on $A_{L L}\left(x_{F}\right)$ in both the $J / \psi$ and $x$ r roduction based on a perturbative QCD approach. 19,20 
REFERENCES

$\S$ Work supported in part by the U.S. Department of Energy, Division of High Energy Physics, Contract W-31-109-ENG-38.

* The E-581/704 Collaboration: Argonne (US), Fermilab (US), Univ. of Iowa (US), Kyoto-Kyoto Sangyo-Kyoto Education-Hiroshima (Japan), LAPP (France), Los Alamos (US), Northwestern Univ. (US), Osaka-Okayama (Japan), Rice Univ. (US), CEN Saclay (France), IHEP Serpukhov (USSR), INFN Trieste- Messina-Udine (Italy), Univ. Occup. Envior. Health (Japan).

1) Grosnick et al., Nucl. Instrum. and Meth. A290 (1990) 269.

2) D. C. Carey et al., Phys. Rev. Lett. 64 (1990) 357.

3) N. Akchurin et a1., Phys. Lett. B229 (1989) 299.

4) B. 2. Kopeliovich and L. I. Lapidus, Yad. Fig. 19 (1974) 218. (Sov. J. Nucl. Phys. 19 (1974) 114).

5) N. H. Buttimore, E. Gotsman, Am. E. Leader, Phys. Rev. D18 (1978) 694.

6) N. H. Buttimore, Proc. 6th Int. Symp. on High Energy Spin Phys. Marseille, J. Soffer, ed., Journal de Physique, 46, C2 (1985) p. 643.

7) S. Sanoff et al., Phys. Rev. Lett. 64 (1990) 995.

8) J. Antille et al., Phys. Lett. B94 (1980) 523.

9) V. D. Apokin et al., Phys. Lett. B243 (1990) 461.

10) D. Sivers, Phys. Rev. D41 (1990) 83; Phys. Rev., to appear.

11) B. E. Bonner et al., Phys. Rev. Lett. 61 (1988) 1918.

12) T. deGrand and H. I. Miettinen, Phys. Rev. D24 (1981) 2419; Phys. Rev. D31 (E) (1.985) 661 .

13) Liang Zuo-liang and Meng Ta-chuisg, Phys. Rev. 42 (1990) 2380; Meng TaChung, Proc. 25th Rencontre de Moriond, Mar. '90, Les Arc, France.

14) M. B. Einhorn and J. Soffer, Nucl. Phys. 274 (1986) 714;

N. S. Craigie, K. Hidaka, M. Jacob, and F. M. Renard, Phys. Reports 99 (1983) 143.

15) E. L. Berger and J. W. Qiu, Phys. Rev. D40 (1988) 788; D40 (RC) (1989) 3128. 
16) S. D. Ellis et a1., Phys. Rev. Lett. 36 (1976) 1263; B. L. Ioffe, Phys. Rev. Lett. 39 (1977) 1589; C. E. Carlson et al., Phys. Rev. D18 (1978) 760; H. Fritzsch, Phys. Lett. 67B (1977) 217; M. Gluck et a1., Phys. Rev. D17 (1978) 2324; L. M. Jones et al., Phys. Rev. D17 (1978) 1782;

V. Berger et al., Z. Phys. C6 (1980) 169; J. H. Kuhn, Phys. Lett. $89 \mathrm{~B}$ (1980) 385; Y. Afek et al., Phys. Rev. D22 (1980) 86.

17) D. A. Bauer et al., Phys. Rev. Lett., 54 (1985) 753; Y. Lemoigne et al., Phys. Lett. 113B (1982) 509 .

18) J. L. Cortes and B. Pire, Phys. Rev. 38 (RC) (1988) 3586.

19) A. P. Contogouris, S.Papadopoulos, and B. Kamal, Phys. Lett. B246 (1990) 523 .

20) M. A. Doncheski and R. Robinett, PSU/TH/67 (1990).

\section{DISCLAIMER}

This report was prepared as an account of work sponsored by an agency of the United States Government. Neither the United States Government nor any agency thereof, nor any of their employees, makes any warranty, express or implied, or assumes any legal liability or responsibility for the accuracy, completeness, or usefulness of any information, apparatus, prorluct, or process disclosed, or represents that its use would not infringe privately owned rights. Reference herein to any specific commercial product, process, or service by trade name, trademark, manufacturer, or otherwise does not necessarily constitute or imply its endorsement, recommendation, or favoring by the United States Government or any agency thereof. The views and opinions of authors expressed herein do not necessarily state or reflect those of the United States Government or any agency thereof. 

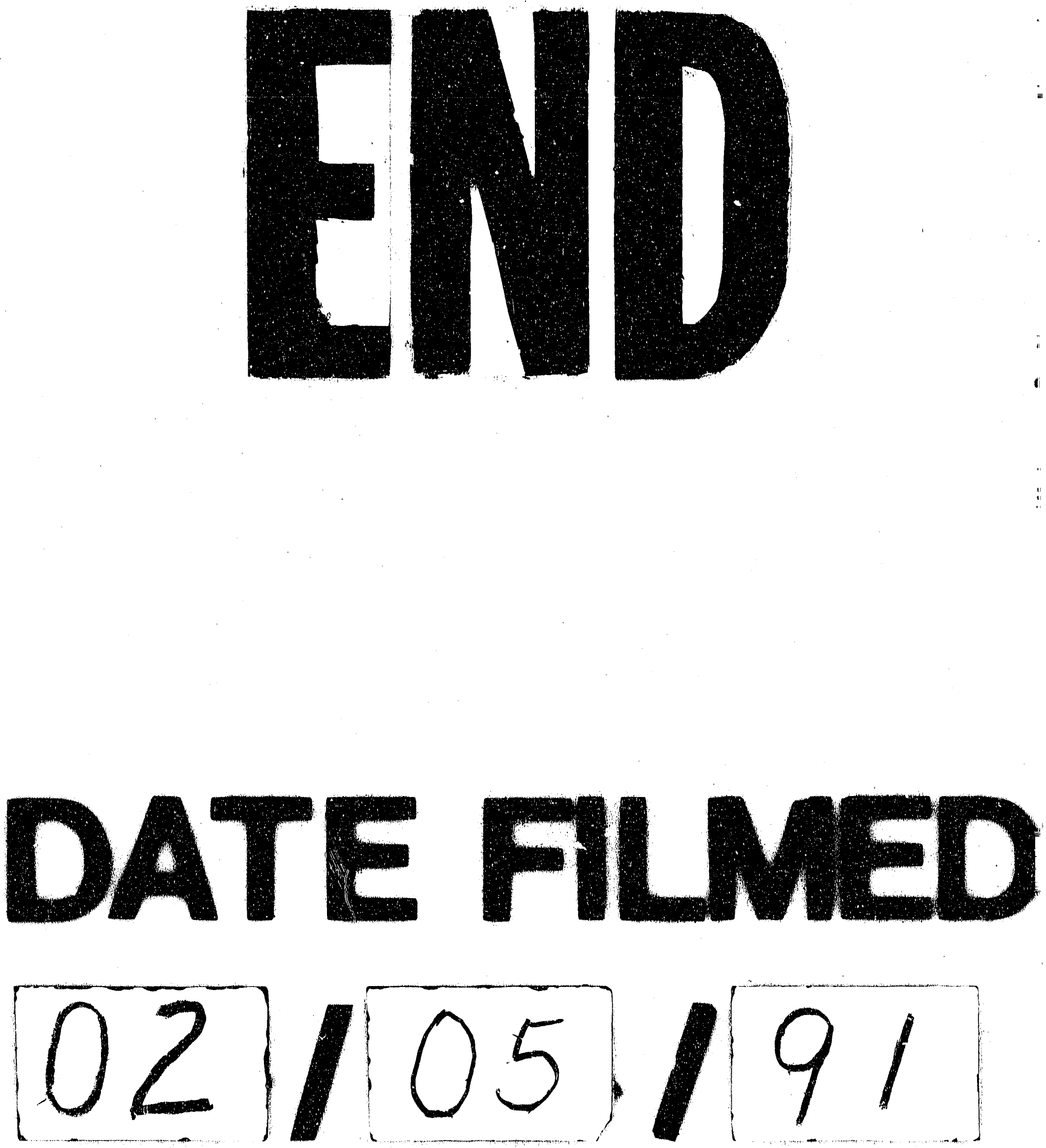